\title{
ASPECTOS CLÍNICOS E TERAPÊUTICOS DAS CEFALÉIAS AGUDAS
}

\author{
CLINICAL AND THERAPEUTIC ASPECTS OF THE ACUTE HEADACHES
}

Getúlio D. Rabello

Mestre em Neurologia pela Faculdade de Medicina da Universidade de São Paulo. Responsável pelo Ambulatório de Cefaléias da Clínica Neurológica do Hospital das Clínicas da Faculdade de Medicina da Universidade de São Paulo.

Correspondência: Rua: Dr. Amâncio de Carvalho no 479 - apto.21 - Vila Mariana - São Paulo - Brasil. CEP: 04012-090; Fax: (011) 572-3674 E-mail-grabell@ibm.net

RABELLO GD Aspectos clínicos e terapêuticos das cefaléias agudas. Medicina, Ribeirão Preto, 30: 458-471, out./dez. 1997.

RESUMO: O autor revisa os aspectos clínicos, diagnósticos e terapêuticos das cefaléias agudas. As cefaléias agudas representam, muitas vezes, uma emergência médica e necessitam de atuação rápida e precisa, com diagnóstico perfeitamente estabelecido. O sucesso do tratamento depende dessas condições. Embora cefaléias primárias sejam importantes diagnósticos diferenciais, cefaléias secundárias ocorrem freqüentemente.

UNITERMOS: Cefaléia. Enxaqueca. Cefaléia Histamínica. Terapêutica.

\section{INTRODUÇÃO}

O Comitê de Taxonomia da International Association for Study of Pain define dor como uma experiência sensorial e emocional desagradável, que é associada a ou descrita em termos de lesões teciduais $^{(1)}$. Cefaléia é definida como todo processo doloroso que ocorre no segmento cefálico. $O$ termo vem do grego $\kappa \varepsilon \phi \alpha \lambda \eta$ (quefalé), que significa cabeça. Portanto, todo paciente afetado por cefaléia sempre relaciona a sintomatologia com algo potencialmente grave. Essa assertiva é particularmente evidente nos pacientes com cefaléias agudas.

Packard ${ }^{(2)}$ interrogou médicos e pacientes a respeito, das expectativas da atuação médica frente a casos de cefaléia. Embora a maioria dos médicos $(66 \%)$ tenham respondido que o desejo dos paciente é a melhora da dor, os pacientes responderam (46\%) que seu maior desejo é uma explicação sobre o quadro que estão apresentando. Isto significa que um dos aspectos mais importantes no atendimento inicial desses pacientes é uma correta explicação dos seus sintomas. Evidentemente que isto é fundamental em qualquer tipo de doença humana, porém, no campo das cefaléias, ele é crítico. Mesmo nas cefaléias agudas, isto é importante, já que muitos pacientes apresentam simplesmente uma agudização de sua patologia crônica.

Em unidades de emergência, tanto portadores de dores agudas como crônicas são freqüentemente atendidos. A dor aguda tem características diferentes das da dor crônica: responde melhor ao tratamento; muitas vezes, representa um sinal de alerta biológico; avaliação diagnóstica e tratamento devem ser rápidos; geralmente, associa-se com muita ansiedade; tem curso auto-limitado e as expectativas em relação ao resultado do tratamento costumam ser muito acentuadas $^{(3)}$.

Em relação ao tempo necessário para definirmos a dor como crônica, não existe consenso na literatura, porém, de maneira geral, a referência é feita para dores com duração total maior que seis meses. 


\section{EPIDEMIOLOGIA}

Uma série de dados epidemiológicos devem ser considerados em relação às cefaléias:

- 5-10\% da população procura médicos, intermitentemente, devido à cefaléia;

- cerca de $40 \%$ dos norte-americanos apresentam cefaléia suficientemente intensa, em algum momento de suas vidas, o que os leva a procurar assistência médica;

- $76 \%$ das mulheres e $57 \%$ dos homens têm, pelo menos, algum tipo de cefaléia por mês;

- 17,6\% das mulheres e 5,7\% dos homens norte-americanos têm enxaqueca.

Embora a maior parte das cefaléias seja crônica, muitos pacientes apresentam-nas de forma aguda, seja em nível ambulatorial ou mesmo em unidades de emergência.

Em nosso meio, alguns dados epidemiológicos nos parecem importantes:

- em ambulatório geral de Clínica Médica, cefaléia representa o terceiro diagnóstico mais comum $(10,3 \%)$, sendo suplantado apenas por infecções de vias aéreas e dispepsias ${ }^{(4)}$;

- em ambulatório de Neurologia, cefaléia representa o mais importante motivo de encaminhamento $(28,54 \%)$, seguido de epilepsia e transtornos mentais $^{(4)}$;

- em levantamento de perto de cem mil atendimentos em unidades de emergência, em cidade do interior do Estado de São Paulo (Taubaté), cefaléias representaram o segundo motivo mais freqüente para atendimento $(28,20 \%$ dos casos $)$, sendo suplantadas apenas por transtornos psiquiátricos (Ferri-de-Barros J.E., comunicação pessoal).

Verificamos, assim, a importância do conhecimento das cefaléias no âmbito da Medicina Geral e, evidentemente, da Neurologia.

\section{ABORDAGEM AO PACIENTE ACOMETIDO DE CEFALÉIA}

Pacientes com cefaléia necessitam de: diagnóstico etiológico, eventual investigação diagnóstica e, por tratar-se de processo álgico, proposição terapêutica sintomática, na primeira avaliação.

Em relação aos aspectos diagnósticos, temos de considerar que estruturas sensíveis à dor devem ser alteradas, para haver sintomatologia dolorosa. Portanto, já no decorrer da história do paciente, temos de ter em mente que, se há cefaléia, alguma estrutura com sensibilidade dolorosa deve estar alterada. $\mathrm{Na}$ cabeça, as estruturas sensíveis à dor são:

- pele, vasos e músculos extracranianos;

- mucosas;

- periósteo;

- dura-máter da base do crânio;

- artérias do polígono de Willis e porções proximais extracerebrais de seus ramos;

- nervos cranianos (V, VII, IX, X) e nervos cervicais.

Existem estruturas não sensíveis à dor, cuja alteração patológica por si só não desencadeia fenômeno doloroso:

- ossos da calota craniana;

- leptomeninges e a maior parte da dura-máter;

- parênquima encefálico e todos os vasos no seu interior.

A história deve ser feita cuidadosamente, já que o diagnóstico, na maior parte das cefaléias, é baseado na anamnese. A história e o exame clínico/neurológico devem conter alguns itens fundamentais; resumidos na Tabela I.

Com esses dados, já podemos estabelecer uma proposição diagnóstica. Porém, é fundamental explicarmos ao paciente qual a nossa impressão. Como colocamos no início desta revisão, a EXPLICAÇÃO é o mais importante item da avaliação de um paciente com cefaléia.

Paciente que procura profissional da área de saúde devido a cefaléia aguda, o faz dentro de uma de quatro situações, que devem ser adequadamente esclarecidas pelo médico:

a) Instalação aguda de cefaléia, dentro de um contexto de cefaléia crônica, sem nenhuma outra doença associada (forte crise de enxaqueca, num quadro crônico e típico desta patologia);

b) Instalação aguda de cefaléia, sem nenhum antecedente prévio de cefaléia (cefaléia aguda de uma hemorragia subaracnóidea, primeira crise de enxaqueca, por exemplo);

c) Cefaléia instalando-se dentro de um quadro clínico em progressão - endocardite infecciosa, com aparecimento de cefaléia associada, de instalação recente, por exemplo;

d) Cefaléia intensa, de caráter crescente, cuja sintomatologia seja suficiente para obrigar o paciente a procurar atendimento médico de urgência - cefaléia da hipertensão intracraniana, por exemplo. 
Tabela I - Elementos da história clínica e do exame físico essenciais para o diagnóstico das cefaléias

I - História clínica

- tipo de dor, localização da dor, intensidade de dor (podemos usar uma pontuação analógica, de 0 a 10, para definirmos a intensidade), irradiação, duração, periodicidade, ritmo, fenômenos físicos e neurológicos acompanhantes, fatores desencadeantes, fatores de melhora e piora, variações da dor baseada em fatores sazonais:

- tratamento atual e passado, e sua efetividade;

- uso de drogas lícitas ou ilícitas, além do uso de cafeína, e sua quantidade;

- história familiar;

- relação com padrão de sono;

- perfil emocional;

- relação da dor com aspectos profissionais do paciente;

- grau de incapacidade decorrente da cefaléia.

\section{II - Exame físico}

- pressão arterial e temperatura;

- palpação, percussão e ausculta da cabeça;

- palpação e ausculta das artérias carótidas e palpação cuidadosa das artérias temporais, superficiais (principalmente em pacientes acima de cinqüenta anos);

- palpação de musculatura cefálica e cervical e dos globos oculares, percussão dos seios de face;

- avaliação de estruturas bucais, pedindo ao paciente para abrir e fechar a boca, palpando estruturas intrabucais e avaliando a articulação temporomandibular;

- otoscopia e percussão das mastóides;

- sistematicamente, avaliar fundo de olho e presença de sinais meníngeos.

Ao atendermos paciente com cefaléia aguda, devemos:

- ter atuação rápida;

- usar o raciocínio clínico;

- evitar transformar a atuação médica num exercício de adivinhação, porém tratar as alternativas diagnósticas prováveis, antes da evolução natural da doença.

\section{ETIOLOGIA}

A International Headache Society ${ }^{(5)}$ definiu as cefaléias em dois tipos: primárias (cefaléias-doença) e secundárias (cefaléias-sintoma). Em inquérito feito com cento e dezoito pacientes, atendidos no Pronto Socorro do Hospital das Clínicas da Faculdade de Medicina da Universidade de São Paulo, em 1996, durante período de três meses, observamos um predomínio discreto de pacientes do sexo feminino (56\%) e uma porcentagem de $58 \%$ de cefaléias secundárias e de $42 \%$ de cefaléias primárias. Portanto, as cefaléias agudas, dos pacientes atendidos habitualmente, em unidades de emergência ou consultórios, têm um padrão diferente do das crônicas, as quais ocorrem, predominantemente, em pacientes do sexo feminino, sendo, geralmente, primárias. A Tabela II nos indica os diagnósticos diferenciais que devemos fazer, baseados na classificação da IHS.

Tabela II - Diagnóstico diferencial das cefaléias segundo a International Headache Society, 1988

Cefaléias primárias

- Enxaqueca

- Cefaléia tipo tensão

- Cefaléia em salvas e hemicrânia paroxística, crônica

- Miscelânea: cefaléia das pontadas, cefaléia de esforço, cefaléia por agente físico (frio), etc.

Cefaléias secundárias

- Cefaléia associada com traumatismo craniano

- Cefaléia associada com doenças vasculares

- Cefaléia associada com doenças intracranianas, não vasculares

- Cefaléia associada com uso ou retirada de substâncias

- Cefaléia associada com infecção não cefálica

- Cefaléia associada com doença metabólica

- Cefaléia ou dor facial associada com várias estruturas (olhos, ouvidos, nariz, garganta, articulação temporomandibular, ossos, coluna cervical)

- Neuralgias cranianas, dor de tronco nervoso e dor por desaferentação

- Cefaléias não classificáveis

\subsection{Enxaqueca}

A enxaqueca representa um complexo sintomatológico caracterizado por crises repetidas com ma- 
nifestações prodrômicas, fenômenos de disfunção neurológica, transitória (aura) e cefaléia (geralmente, de caráter pulsátil, com alterações neurovegetativas, fotofobia, fonofobia, osmofobia). Qualquer uma dessas manifestações pode faltar, inclusive a cefaléia.

Schlake et al. ${ }^{(6)}$ chamam a atenção para a possibilidade de lesões intracranianas simularem crises de enxaqueca. Afirmam que, embora os critérios operacionais da classificação da International Headache Society ${ }^{(5)}$ tenham sido importantes no diagnóstico das cefaléias, não permitem uma inequívoca discriminação entre cefaléias primárias e secundárias. Segundo a opinião dos autores, seria interessante uma investigação em pacientes com enxaqueca, nas seguintes eventualidades:

- associação entre manifestações enxaquecosas e hemiparesias transitórias;

- início tardio dos sintomas;

- mudança das características da dor e dos sintomas acompanhantes.

As situações agudas, nas quais o diagnóstico de enxaqueca pode ser feito, são discriminadas a seguir:

a) Aura enxaquecosa, sem cefaléia: nesta situação, episódio isquêmico, transitório é uma consideração diagnóstica; geralmente, a aura enxaquecosa é mais prolongada, e as manifestações são mais estereotipadas, existindo progressão das manifestações, não respeitando território arterial; eventualmente, o diagnóstico diferencial pode ser difícil;

b) Estado enxaquecoso: crise de enxaqueca, durando mais de setenta e duas horas, entremeada com eventuais períodos de acalmia, inferiores à quatro horas.

\subsection{Cefaléia tipo tensão}

Carateriza-se por cefaléia de caráter em aperto, ora nucal, ora frontal, e, muitas vezes, holocraniana, geralmente, não piorando com a atividade física. Fenômenos neurovegetativos não costumam ser encontrados. Pode ter caráter episódico ou crônico.

\subsection{Cefaléia em salvas e hemicrânica paroxís- tica, crônica}

A cefaléia em salvas (episódica ou crônica) caracteriza-se por crises de cefaléia unilateral, cruciante, de localização frontotempororbitária, com duração em torno de trinta a noventa minutos, ocorrendo, muitas vezes, em horários determinados, geralmente, durante a madrugada. Semiptose, lacrimejamento, rinorréia, hiperemia conjuntival, miose podem ocorrer. As crises costumam ocorrer em determinados períodos do ano, geralmente com ciclos de oito a doze semanas (fazem exceção os casos crônicos). Quando as crises têm curta duração, ocorrendo muitas vezes ao dia e em pessoa do sexo feminino, devemos pensar em hemicrânia paroxística, crônica, que tem resposta terapêutica excelente à Indometacina. Na modalidade episódica da hemicrânia paroxística, períodos de remissão prolongados podem ser observados. Frente a este grupo de entidades nosológicas, uma série de diagnósticos diferenciais devem ser afastados. Tais possibilidades são catalogados a seguir.

a) Síndrome SUNCT - Caracteriza-se por paroxismos de dor, com duração entre cinco e duzentos e cinqüenta segundos, em grande número (5-6/hora), unilaterais, com caráter em queimação, ou choque elétrico, acompanhada por hiperemia conjuntival, lacrimejamento, rinorréia e sudorese frontal. É mais freqüente em homens e tem resposta terapêutica insatisfatória a, praticamente, todos os medicamentos usados no tratamento das crises de cefaléia em salvas, hemicrânias paroxísticas e neuralgias $^{(7)}$. Pode, eventualmente, ser manifestação sintomática de malformações arteriovenosas, de fossa posterior ou de vasculite venosa, orbitária ${ }^{(8,9)}$;

b) Neuralgia trigeminal;

c) Sinusite esfenoidal;

d) Dissecção de artéria carótida, interna;

e) Arterite temporal;

f) Tumores de fossa média e posterior;

g) Patologia orbitária;

h) Síndrome de Tolosa-Hunt.

\subsection{Miscelânea}

Nesse grupo, encontramos várias modalidades de cefaléia. Destacamos as chamadas cefaléias de esforço. Pascual et al. ${ }^{(10)}$ avaliaram pacientes com cefaléia ligada à tosse, ao exercício ou à atividade sexual e observaram $42 \%$ com doenças associadas, como a malformação de Chiari, tipo I - principalmente nos casos ligados à tosse - e a hemorragia subaracnóidea - nos casos de cefaléia ligada ao exercício e à atividade sexual. Quando a cefaléia de esforço se apresenta como quadro essencial, a medicação de escolha é a Indometacina. 


\subsection{Cefaléia associada com traumatismo craniano}

Pacientes acometidos por cefaléia, instalando-se em período inferior a duas semanas, após normalização do estado de consciência, eventualmente alterado por traumatismo craniano, podem apresentar o que é denominado cefaléia pós-traumática. Esses pacientes devem ser investigados, porque complicações do traumatismo podem ocorrer alguns dias após o acidente: hematoma subdural, crônico; hemorragia subaracnóidea, traumática; fístula liquórica, com hipotensão liquórica. A cefaléia pós-traumática pode ter características clínicas de cefaléia tipo enxaqueca, tensão ou em salvas.

\subsection{Cefaléia associada com doenças vasculares}

O aparecimento de cefaléia abrupta, acompanhada de sinais focais neurológicos, persistentes, determina um diagnóstico presuntivo de doença vascular cerebral, como acidente vascular, cerebral, isquêmico ou hemorrágico. Algumas considerações são importantes, já que o tópico demanda importantes diagnósticos diferenciais.

a) Hemorragia subaracnóidea (HSA): a ruptura de um aneurisma ou malformação arteriovenosa determina cefaléia intensa, acompanhada de náuseas e vômitos, muitas vezes com alterações do estado de consciência e presença de sinais meníngeos. $\mathrm{O}$ quadro clássico pode estar ausente, determinando dificuldades diagnósticas. Weir B. ${ }^{(11)}$ refere que $10 \%$ dos pacientes com HSA não se queixam de cefaléia e $8 \%$ manifestam cefaléia discreta, com incremento progressivo. Cerca de $40 \%$ dos pacientes apresentam a chamada hemorragia sentinela, que são pequenos sangramentos que precedem a ruptura significativa do aneurisma. A dor pode ser unilateral, de localização hemicrâniana ou hemifacial. A tomografia computadorizada cerebral (TCC) pode ser normal e somente a punção liquórica pode definir o diagnóstico. Cerca de $50 \%$ dos pacientes, apesar de procurarem assistência médica, são incorretamente diagnosticados. Outra situação é a chamada "cefaléia em trovoada" (thunderclap headache), que foi descrita por Day \& Raskin $^{(12)}$. Trata-se de situação em que o paciente apresenta cefaléia intensa, com náuseas e vômitos, em tudo simulando uma HSA. Porém TCC e líquido cefalorraquidiano (LCR) são normais. Os autores preconizam a realização de angiografia cerebral, já que observaram a presença de aneuris- mas em alguns de seus casos. Estudos posteriores trouxeram dúvidas em relação a tal conduta. Não tem sido norma a realização de angiografia convencional, após TCC e LCR normais. Em caso de dúvida, contamos, hoje, com métodos não invasivos de investigação, como a angiografia por ressonância magnética.

b) A arterite temporal ocorre, principalmente, em pacientes idosos. Sua instalação pode ser progressiva, antes do aparecimento de sintomas gerais mais típicos da doença (dores musculares, febrícula, claudicação da mandíbula, perda de peso, claudicação de extremidades e déficit visual). Qualquer paciente, acima de cinqüenta anos, com cefaléia de início recente, deve ser submetido a essa consideração diagnóstica, com eventual realização de exames gerais (hemograma, hemossedimentação, proteína C-reativa, eletroforese de proteínas séricas).

c) Dissecção arterial tem se transformado em importante causa de cefaléias agudas. Quando a dissecção ocorre no território da artéria carótida, dores na região cervical, irradiando-se para o hemicrânio ipsilateral, podem ocorrer, seguidas, após alguns dias, de sinais focais ipsilaterais (amaurose fugaz) ou contralaterais (alterações motoras, sensitivas ou de linguagem). Cefaléia lateralizada, principalmente se acompanhada de síndrome de Claude Bernard-Horner, ipsilateral, deve ser investigada.

d) Trombose de grandes seios venosos pode manifestar-se exclusivamente com cefaléia, com características de hipertensão intracraniana. Alterações no exame de fundo de olho podem faltar, dificultando o diagnóstico.

e) Em relação às cefaléias em pacientes com hipertensão arterial, a maior parte deles apresenta outras doenças: enxaqueca, cuja sintomatologia é piorada pela hipertensão arterial, hemorragia subaracnóidea, encefalopatia hipertensiva, doença cerebrovascular. Portanto, apenas quando afastarmos completamente essas possibilidades, é que poderemos dizer que se trata de cefaléia diretamente ligada à hipertensão arterial. Além disso, alguns pacientes portadores de feocromocitoma podem apresentar cefaléia de curta duração. Em cerca de $70 \%$ das vezes, tal tipo de cefaléia tem duração inferior a uma hora, podendo durar entre um a três minutos. Pode ocorrer associada à alguma atividade física ou à micção, como no caso do feocromocitoma vesical. A presença de sintomas ligados à liberação de catecolaminas (ansiedade, sudorese, palpitação), pode facilitar o diagnóstico. 


\subsection{Cefaléia associada com doenças intracrania- nas, não vasculares}

As cefaléias que acompanham doenças infecciosas do sistema nervoso central, geralmente, aparecem num contexto de febre e sinais meníngeos. Quanto aos tumores, com o advento dos exames de imagem, o diagnóstico é feito muito precocemente, antes que a cefaléia clássica matutina venha a ocorrer. Forsyth $\&$ Posner $^{(13)}$ observaram que, muitas vezes, a cefaléia ligada aos tumores cerebrais tem aspectos clínicos que lembram enxaqueca ou cefaléia tipo tensão. Náuseas, vômitos, exame neurológico anormal ou mudança significante do padrão de cefaléia prévia sugerem a possibilidade de um processo expansivo intracraniano.

Algumas observações adicionais nos parecem importantes.

a) Mathew et al. ${ }^{(14)}$ observaram que $14 \%$ dos pacientes portadores de cefaléias crônicas resistentes, sem alterações no exame neurológico, inclusive no exame de fundo de olho, apresentavam hipertensão intracraniana à punção liquórica. $\mathrm{O}$ diagnóstico de pseudotumor cerebral deve ser aventado em tal situação;

b) A cefaléia da hipotensão liquórica pode ocorrer espontaneamente, embora seja mais freqüente após punção lombar. O diagnóstico é sugerido pela alteração da dor com mudanças de posição, com ou sem sintomas associados. Embora a confirmação possa ser feita pela medida de pressão liquórica, durante punção lombar, ela às vezes, pode ser normal. A ressonância magnética pode mostrar padrão sugestivo, caracterizado por realce meníngeo difuso, quando da infusão de gadolíneo ${ }^{(15)}$.

\subsection{Cefaléia associada com uso ou retirada de substâncias}

A relação entre cefaléia e substâncias químicas é conhecida há muito tempo. No entanto, o fato de as drogas utilizadas para o tratamento poderem ser também fator importante no seu desencadeamento, embora possa parecer fato de interesse recente, já havia chamado a atenção de Peters \& Horton, em 1951 ${ }^{(16)}$ : "o fenômeno de rebote ocorre - a cefaléia da abstinência começa e mais Tartarato de Ergotamina deve ser ingerido, para combatê-la. Um círculo vicioso se desenvolve e mais e mais Tartarato de Ergotamina é necessário, para produzir o efeito desejado".
Vários são os mecanismos pelos quais as drogas geram cefaléia ${ }^{(17)}$.

a) Exposição aguda a uma substância: nitratos/nitritos, glutamato monossódico; monóxido de carbono e óxido de nitrogênio - estes relacionados aos índices de poluição atmosférica; álcool, adoçantes artificiais - aspartame; tóxicos - cocaína, maconha; medicações - Hidralazina, bloqueadores de canais de cálcio, dipiridamol, fenotiazínicos, teofilinas, corticosteróides, simpatomiméticos como Efedrina, inibidores de MAO, indometacina, ranitidina e cimetidina, Trimetropin-sulfametoxazol;

b) Suspensão de substância de uso agudo: o exemplo típico é a cefaléia da ressaca, relacionada ao álcool;

c) Cefaléia associada a substâncias por mecanismo incerto: o exemplo típico é cefaléia ligada ao uso de anticoncepcionais orais;

d) Cefaléia induzida pela exposição crônica e retirada de substância de uso crônico: aqui temos as cefaléias induzidas por drogas, pelo abuso de analgésicos e cafeína. Habitualmente, esta cefaléia leva a sintomatologia mais crônica.

\subsection{Cefaléia associada com infecção não cefálica}

A presença de febre associada com cefaléia sempre nos leva à possibilidade de infecção do sistema nervoso central. Os quadros de encefalite são facilmente eliminados, já que as manifestações de envolvimento encefálico são evidentes - alterações de estado de consciência, crises convulsivas, sinais neurológicos focais. O diagnóstico diferencial mais importante é o com relação às meningites. Nas meningites bacterianas agudas, geralmente, os sinais meníngeos são evidentes e o diagnóstico não oferece dificuldade. As maiores dificuldades ocorrem nas chamadas meningites linfomonocitárias agudas e nas meningites crônicas (tuberculose, fungos, por exemplo). Não existem regras claras de conduta, porém uma assertiva que julgamos fundamental é que, "na dúvida, faça-se uma punção liquórica".

Uma situação que vem se tornando cada vez mais freqüente, em unidades de emergência e mesmo ambulatoriais, é a do paciente portador de infecção pelo vírus HIV, que apresenta cefaléia. Goldstein ${ }^{(18)}$ observou que, embora cefaléias primárias possam ocorrer, o sintoma pode representar indício de manifestações infecciosas ou neoplásicas do sistema nervoso central. Quando a cefaléia é difusa e associada 
com náuseas, existe maior possibilidade de tratar-se de cefaléia secundária. Dentre essas, a mais importante como diagnóstico presuntivo é a neurocriptococose. Portanto, todo paciente com infecção HIV e cefaléia, mesmo na ausência de evidências mais claras de infecção do sistema nervoso central, deve ser submetido à investigação, com TCC e LCR.

\subsection{Cefaléia associada com doença metabólica}

Hipóxia, hipoglicemia e alterações causadas por diálise são as causas mais frequientes nessa situação. Quanto à hipóxia, devemos chamar a atenção para o fato de que cefaléia do despertar pode ocorrer em casos de apnéia do sono e muitos pacientes com cefaléia em salvas e outras formas de cefaléia crônica apresentam apnéia do sono ${ }^{(19)}$.

Pacientes que realizam diálise podem apresentar cefaléia, geralmente com duração inferior a vinte e quatro horas. Essa cefaléia desaparece com a mudança dos parâmetros da diálise. Eventualmente, complicações do procedimento, como o hematoma subdural crônico, podem se manifestar com cefaléia.

\subsection{Cefaléia ou dor facial, associada com estru- turas (olhos, ouvidos, nariz, garganta, arti- culação temporomandibular, ossos, coluna cervical)}

As cefaléias com componente facial de dor devem ser investigadas, porque, habitualmente, são secundárias. Destacamos, a seguir, as principais cefaléias desse tipo.

a) Cefaléias relacionadas à coluna cervical: lesões estruturais da região podem causar cefaléia. O desencadeamento da dor com movimentação cervical pode indicar sua origem. Sjaastad et al. ${ }^{(20)}$ descreveram uma cefaléia, considerada primária, denominada cefaléia cervicogênica. Suas características estão descritas abaixo:

- dor unilateral (occipital ou frontal);

- ataques infreqüentes e de longa duração;

- intensidade moderada;

- associação com envolvimento cervical;

- fotofobia, fonofobia, náuseas, vômitos, tonturas, borramento visual, lacrimejamento, hiperemia ocular, ipsilateral;

- zonas de gatilho, presentes na palpação da região cervical;

- melhora com bloqueio anestésico do nervo occipital maior e/ou menor.
Existe muita controvérsia, na literatura, sobre a entidade, principalmente em relação a sua situação como entidade primária ou secundária, associada a fatores miofaciais. Porém, o que devemos ter em mente é que a avaliação da coluna cervical deve fazer parte da semiologia de qualquer paciente com cefaléia.

b) Sinusite esfenoidal: processos inflamatórios e infecciosos do seio esfenoidal podem gerar cefaléia de localização variável, às vezes, profunda, em aperto e, eventualmente, no vértex. Secreção nasal pode não ser evidente e, muitas vezes, pacientes podem ser considerados como acometidos por cefaléia primária, geralmente, cefaléia tipo tensão episódica. Febre pode levar à suspeita de quadro meníngeo. Secreção na orofaringe pode levar à suspeita diagnóstica. Tomografia computadorizada pode auxiliar no diagnóstico, devido à dificuldade observada com o RX-simples, de crânio. Lesões profundas, de outra natureza, como os tumores de rinofaringe posterior, podem levar a dificuldades diagnósticas semelhantes.

\subsection{Neuralgias cranianas, dor de tronco ner- voso e dor por desaferentação}

As dores com caráter neurálgico têm duração curta, em choque, e são restritas ao território de um nervo específico. As entidades nosológicas mais importantes são a neuralgia trigeminal e a neuralgia glossofaríngea. Afecções de tronco nervoso (como a dor relacionada ao herpes zoster) tem sempre a característica de restringir-se ao território do nervo afetado.

Devemos chamar a atenção para duas entidades, neste capítulo:

- Dor facial atípica (odontalgia atípica): dor facial persistente, que não tem as características das neuralgias cranianas, e não está associada a sinais físicos ou causa orgânica demonstrável. A dor costuma ser diária e persiste a maior parte do dia. Costuma iniciar-se numa área limitada da face, porém, posteriormente, irradia-se para área mais ampla. Tem localização profunda e é mal definida. Às vezes, pode iniciar-se após cirurgia ou trauma envolvendo face, dentes ou gengiva, porém persiste sem qualquer causa demonstrável. É diagnóstico de exclusão e que só deve ser feito após exaustiva investigação. Capobianco $^{(21)}$ chama a atenção para a realização de RX de tórax, em todo paciente com dor facial atípica, pela possibilidade de associação com neoplasia pulmonar. 
- Síndrome de Eagle: caracterizada por dor de caráter contínuo ou paroxístico, sendo devida ao alongamento do processo estilóide (cujo tamanho deve ser maior que $4 \mathrm{~cm}$, incluindo o processo em si e ligamentos). A dor costuma ter localização parietal, ocular, facial, cervical ou auricular, e geralmente se associa com sensação de corpo estranho na garganta. A dor pode ser desencadeada pelos atos de deglutir, falar, cantar e bocejar. O diagnóstico pode ser feito pela palpação externa da região cervical anterior e interna da boca. A confirmação do quadro se faz através de radiografias simples.

\subsection{Cefaléias não classificáveis}

Muitos pacientes apresentam quadros não classificáveis. Nessa situação, repetição da anamnese, exame clínico e neurológico é fundamental. Investigação deve ser feita no sentido de afastar doenças secundárias. Esses pacientes devem ser reavaliados periodicamente, no sentido de ser estabelecido diagnóstico etiológico preciso.

Tabela III - Modalidades de cefaléia, não consideradas na classificação da International Headache Society, 1988

1 - Cefaléias indometacina-responsivas

- Hemicrânia, paroxística, crônica

- Hemicrânia, paroxística, episódica

- Cefaléia idiopática das pontadas

- Cefaléia de esforço

- Hemicrânia, contínua

2 - Cefaléia crônica diária

- Enxaqueca transformada

- Cefaléia tipo tensão, crônica

- Cefaléia persistente, diária, nova

- Hemicrânia, contínua

3 - Cefaléia relacionada a patologias cervicais

- Cefaléia por alterações estruturais cervicais

- Cefaléia cervicogênica

4 - Cefaléia hípnica

5 - Síndrome SUNCT

\subsection{Outras modalidades de cefaléia}

Nos parágrafos anteriores, Tabela III descrevemos algumas formas de cefaléia não definidas na classificação da IHS. Enumeramos, abaixo, aquelas que não foram descritas previamente.

Hemicrânia contínua - Estritamente unilateral, de caráter contínuo, com poucos sinais e sintomas clínicos e autonômicos associados (quando comparada com a cefaléia em salvas e hemicrânia paroxística, crônica), sem mecanismos de desencadeamento e resposta terapêutica excelente à indometacina. A dor pode ter caráter de aperto, pulsátil e, mesmo, de pontadas. Trata-se de diagnóstico de exclusão, sendo necessária investigação para afastar doenças associadas ${ }^{(22)}$.

Cefaléia hípnica - Ocorre em pacientes idosos (acima de sessenta e cinco anos). Geralmente, acorda o paciente do sono no mesmo horário, muitas vezes, durante um sonho. Dura em torno de trinta a sessenta minutos, é holocraniana, pulsátil, com náuseas e sem outros sintomas autonômicos associados. Tem boa resposta terapêutica ao Carbonato de Lítio, na dose de 300 a $600 \mathrm{mg}$, ao deitar ${ }^{(23)}$.

\section{CEFALÉIA - AVALIAÇÃO INICIAL}

Devemos formular para nós mesmos algumas perguntas, quando atendemos um paciente com cefaléia.

\subsection{Dores agudas e crônicas são diferentes?}

Freqüentemente, médicos, que atendem pacientes com dor crônica, sentem-se frustrados, irritados e muitas vezes têm incertezas, irritação e rejeição em relação aos mesmos. É necessário entender que, embora tais reações sejam normais, podem interferir na relação médico-paciente e, evidentemente, comprometer o tratamento. A perfeita compreensão de tais reações pode ajudar a guiar o médico, no manejo de pacientes com dor crônica.

Pacientes com dor crônica, mesmo com algumas manifestações agudas, habitualmente, não têm aspecto de sofrimento evidente. Isto não significa que estejamos frente a quadro de simulação. Até prova em contrário, o paciente com dor, e cefaléia é simplesmente uma modalidade de dor, deve ser corretamente avaliado e o diagnóstico preciso deve ser feito. $\mathrm{O}$ fato de haver resposta da dor à medicações do tipo placebo não significa, necessariamente, que a dor não seja real, pois o efeito placebo ocorre freqüentemente em qualquer modalidade de dor e, particularmente, nas cefaléias. 


\subsection{O padrão de cefaléia pode nos dar pistas sobre a etio- logia?}

As cefaléias podem ser classificadas, em relação à sua evolução temporal, em vários tipos: cefaléia aguda, emergente; cefaléia aguda, recorrente; cefaléia crônica, progressiva e cefaléia crônica, não progressiva. Na figura 1, podemos observar como a dor evolui nos diferentes tipos.

Cada padrão de cefaléia admite determinados tipos de cefaléia como mais comuns; como mostra a Tabela IV.

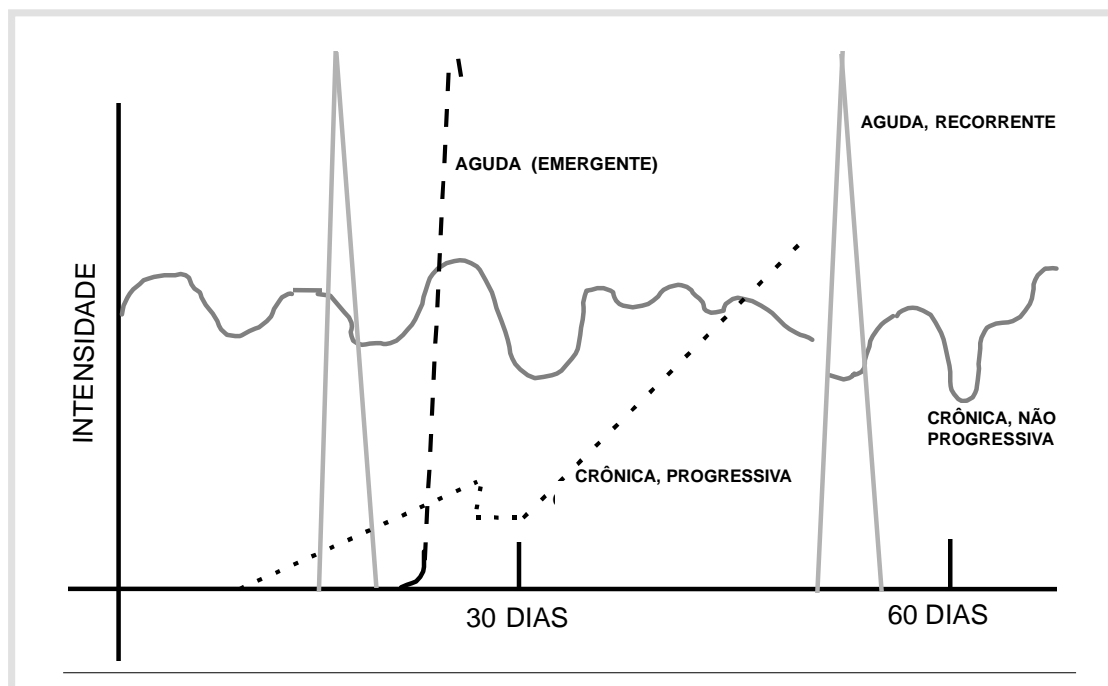

Figura 1 - Classificação temporal das cefaléias

\section{Tabela IV - Correlação entre o padrão clínico e o diagnóstico da cefaléia}

Cefaléia aguda emergente - nesse caso, uma cefaléia não habitual ocorre, em paciente sem cefaléia crônica, e os principais diagnósticos diferenciais são:

- primeira crise de enxaqueca,

- hemorragia intracraniana, aguda,

- hipertensão arterial, aguda e grave,

- infecção sistêmica,

- sinusite e celulite,

- glaucoma agudo,

- dissecção arterial, aguda,

- hidrocefalia,

- traumatismo craniano,

- meningite e encefalite,

- trombose venosa, cerebral,

- neurite óptica,

- doença cerebrovascular, isquêmica,

- arterite.

Cefaléia aguda recorrente - cefaléia aguda, em paciente que já apresentou episódios semelhantes no passado, evidentemente, toda cefaléia aguda recorrente foi, em determinado momento, uma cefaléia aguda emergente, e os principais diagnósticos diferenciais são:

- enxaqueca,

- cefaléia em salvas (rara em crianças),

- neuralgia trigeminal (raro em crianças),

- hemorragia subaracnóidea (principalmente nas malformações arteriovenosas),

- doença cerebrovascular, isquêmica,

- hidrocefalia intermitente (neurocisticercose, cisto colóide do III ventrículo),

- feocromocitoma (podendo determinar crises de cefaléia episódica, de curta duração).
Cefaléia crônica progressiva - com intensidade crescente, ocorre, indicando, geralmente, doença grave associada, e os principais diagnósticos diferenciais são:

- hematoma subdural,

- tumor,

- abscesso cerebral,

- pseudotumor cerebral,

- arterite temporal,

- doenças da coluna cervical,

- doenças da transição occipitocervical,

- sinusite,

- hipotensão liquórica,

- cefaléia associada a drogas,

- infecções do sistema nervoso central,

- anormalidades metabólicas

- hemicrânia contínua (cefaléia primária).

Cefaléia crônica não progressiva - que tem duração de semanas, meses ou anos, com flutuações na sua intensidade. Embora raramente possa indicar alteração estrutural, de forma geral não apresenta doença grave associada, embora represente o maior problema nos serviços especializados em cefaléia, e os principais diagnósticos diferenciais são:

- estados psiquiátricos,

- cefaléia tipo tensão,

- enxaqueca transformada,

- uso abusivo de analgésicos,

- cefaléia crônica, diária, reincidente,

- tumor,

- problema oftalmológico,

- espondilose cervical,

- pseudotumor cerebral. 
5.3. Quais os erros mais freqüentes, observados na avaliação das cefaléias agudas, nas unidades de emergência?

Existem algumas situações em que equívocos diagnósticos são cometidos e que podem levar a graves complicações para os pacientes. As principais situações, em que tal ocorre, são:

- associação entre cefaléia e alcoolismo, na qual, traumatismos cranioencefálicos não são detectados, considerando-se a situação simplesmente como "cefaléia da ressaca";

- cefaléia associada com hipertensão arterial, na qual hemorragia subaracnóidea não é detectada;

- cefaléia em idosos, na qual arterite temporal não é lembrada;

- paciente idoso, com cefaléia e rigidez cervical, erroneamente interpretada como sendo devida à artrose cervical e que, na verdade, pode representar meningite associada;

- odontalgia, às vezes, devida à pulpite, erroneamente diagnosticada como neuralgia essencial do trigêmeo;

- glaucoma não diagnosticado;

- sinusite esfenoidal, interpretada como cefaléia primária;

- paciente portador de cefaléia, muitas vezes, devida a patologia estrutural importante, que recebeu medicações sedativas e que tem sua alteração de consciência relacionada à medicação usada e não à alteração estrutural causadora (hemorragia subaracnóidea, meningoencefalite, hematoma subdural crônico, por exemplo).

\subsection{Quando devemos partir para investigação la- boratorial e/ou radiológica?}

Evidentemente, a avaliação clínica pode permitir um diagnóstico e uma orientação segura, na maioria das situações. Temos utilizado, como critério de investigação, as seguintes situações:

- história não sugestiva de cefaléia primária;

- alterações no exame clínico e/ou neurológico;

- cefaléia de início agudo, recente e de forte intensidade (cefaléia aguda, emergente);

- mudança de característica de cefaléia crônica;

- cefaléia de esforço;

- cefaléia com componente facial de dor;

- cefaléia associada à infecção pelo vírus HIV;

- cefaléia em salvas;
- cefaléia noturna, de início recente;

- cefaléia com componente miofacial importante;

- cefaléia na infância (pelas dificuldades na anamnese nestes pacientes);

- estado enxaquecoso resistente à medicação (principalmente no sentido de afastar doenças inflamatórias ou infecciosas do espaço subaracnóideo).

\subsection{Existe um exame mais eficiente na investi- gação?}

A investigação vai depender da hipótese diagnóstica formulada. Assim, se suspeitamos de arterite temporal, a avaliação da velocidade de hemossedimentação é mais importante que uma ressonância magnética (RNM) de crânio. Nas situações em que não temos nenhuma hipótese clara, e o exame do paciente é normal, a RNM é o melhor exame, já que consegue demonstrar processos da sela túrcica, doenças de seios venosos intracranianos e alterações da transição occipitocervical, de forma mais eficiente ${ }^{(24)}$.

\subsection{Realizada investigação de paciente com ce- faléia, o achado de alterações nos exames subsidiários determina, definitivamente, $o$ diagnóstico?}

Após a verificação de alterações, temos de tentar estabelecer correlações entre as referidas alterações e o quadro clínico do paciente. Assim, o achado de espessamento de mucosa, nos seios da face, não significa, necessariamente, que sinusite seja a causa da cefaléia, assim como a presença de calcificações intracranianas não necessariamente significa que neurocisticercose é a causa da cefaléia.

\section{TERAPÊUTICA DAS CEFALÉIAS AGUDAS}

O tratamento dependerá da modalidade de cefaléia diagnosticada. Assim, descreveremos, abaixo, os tratamentos efetuados nas várias formas de cefaléias primárias e secundárias.

\subsection{Enxaqueca}

Habitualmente, o paciente, que procura a unidade de emergência devido à enxaqueca, encontra-se há várias horas com a crise, e já utilizou medicamentos via oral, sem sucesso, seja por ineficácia dos mesmos, seja pelas náuseas e vômitos associados. É muito 
importante uma adequada avaliação do estado de hidratação do mesmo, havendo necessidade de correção, se for o caso. Uma série de medicações pode ser usada; como as discriminadas na Tabela V.

\section{Tabela V - Tratamento farmacológico da enxa-} queca

- Metoclopramida, 10 mg + Dipirona EV.

- Antiinflamatórios não hormonais IM (Diclofenaco, Cetoprofeno).

- Metoclopramida, $10 \mathrm{mg}+$ Dipirona EV + Dexametasona $8 \mathrm{mg} \mathrm{EV}$.

- Sumatriptan, $6 \mathrm{mg} \mathrm{SC}$ (pode ser repetido uma vez).

- Meperidina + Antieméticos IM (principalmente, em mulheres grávidas).

- DHE 1mg + Prometazina 50mg ou Clorpromazina $50 \mathrm{mg}$ IM.

- Metoclopramida, 10 mg EV + DHE 0,5-0,75 mg EV.

- Metoclopramida, $10 \mathrm{mg} \mathrm{EV} \mathrm{+} \mathrm{DHE} \mathrm{0,5-0,75} \mathrm{mg}$ $\mathrm{EV}+$ Dexametasona, $8 \mathrm{mg}$ IM.

Em nosso meio, não contamos com DHE (Diidroergotamina injetável), que apresenta eficácia semelhante à do Sumatriptan.

$\mathrm{O}$ uso de agentes antidopaminérgicos tem eficácia no tratamento de crises de enxaqueca resistentes, inclusive na situação denominada "estado enxaquecoso". Em recente revisão, Peroutka ${ }^{(25)}$ enfatiza as evidências, cada vez maiores, de hiperatividade dopaminérgica, na enxaqueca, que pode ser controlada de forma muito eficiente por agentes farmacológicos. Vários esquemas podem ser usados, como os catalogados na Tabela VI.

O bloqueio do nervo occipital maior com Lidocaína, sem vasoconstritor pode ser recurso invasivo no tratamento de crises resistentes de enxaqueca. A explicação para sua atuação é que, diminuindo a atividade aferente, proveniente das raízes cervicais rostrais - que constituem o nervo grande occipital que se projetam para o núcleo do trato espinal do trigêmeo, importante núcleo de integração da dor cefálica, existiria diminuição da sua atividade. O nervo é localizado na linha nucal superior, no meio de uma linha virtual entre a mastóide e a protuberância occipital. Cerca de $1 \mathrm{ml}$ de Lidocaína, a 2\%, sem vasoconstritor, é injetado nessa região, sendo $0,6 \mathrm{ml}$ perpendicularmente, e $0,6 \mathrm{ml} 5^{\circ}$, lateralmente, e $5^{\circ}$, medialmente.
Tabela VI - Terapêutica antidopaminérgica da enxaqueca

- Clorpromazina - 0,1 mg/kg/EV, dissolvido em $500 \mathrm{ml}$ de Soro Fisiológico, em infusão rápida (trinta minutos) pode ser feito. Esta infusão pode ser repetida três vezes em vinte e quatro horas. Cuidado especial deve ser tomado em relação ao controle da pressão arterial (hipotensão arterial). Eventualmente, o uso de $1 \mathrm{mg} / \mathrm{kg} / \mathrm{IM}$ pode ser usado. Outra forma de administração é preparar solução de Clorpromazina em Soro Fisiológico, na concentração de $5 \mathrm{mg} / \mathrm{cc}$ e aplicar $1 \mathrm{cc}(5 \mathrm{mg})$ a cada dez minutos, parando, quando a cefaléia tiver cedido, ou o máximo de $25 \mathrm{mg}$ tiver sido aplicado. É conveniente infundir $500 \mathrm{ml}$ de solução fisiológica previamente aos primeiros $5 \mathrm{mg}$ aplicados ${ }^{(26)}$. Bastos et al. ${ }^{(27)}$ demonstraram a eficácia da Clorpromazina em gotas (10 gotas de solução a 4\%), por via sublingual, no tratamento de crises resistentes de cefaléia.

- Metoclopramida - $10 \mathrm{mg}$ EV, três vezes ao dia, pode ser usado, como já foi exposto previamente. A Metoclopramida, além de sua ação antiemética, pode ter ação analgésica, associada, em crises de enxaqueca.

- Haloperidol - 5 mg EV.

- Proclorperazina - 10 mg EV.

- Domperidona - encurta o período de uma crise de enxaqueca. Como este agente antidopaminérgico não penetra pela barreira hematoencefálica, provavelmente, muitos sintomas da crise de enxaqueca advêm da ativação de receptores dopaminérgicos periféricos.

Uma difícil situação é encontrada em pacientes que procuram atendimento de urgência com aura enxaquecosa prolongada, persistindo isoladamente ou com a fase álgica já instalada. O uso de vasoconstritores, como os ergóticos, deve ser evitado (embora as evidências de diminuição do fluxo sangüíneo cerebral pelos mesmos sejam pequenas). Verapamil injetável ou Nifedipina sublingual (10 mg) têm sido usados, porém com resultados não definidos, na literatura. $\mathrm{O}$ uso de analgesia e sedação, induzindo o sono, parece ser a terapêutica mais efetiva. Eventualmente, inalação com Nitrato de Amila ou Isoproterenol pode ser feita ${ }^{(26)}$.

\subsection{Cefaléia tipo tensional}

A cefaléia do tipo tensional (CTT), episódica deve ser controlada com analgésicos comuns e, eventualmente, com medicações sedativas leves (diazepínicos, por via oral). A CTT, crônica tem seu tratamento principalmente em unidades ambulatoriais. 


\subsection{Cefaléia em salvas}

A cefaléia em salvas (CS), seja episódica ou crônica, representa forma extremamente intensa de cefaléia primária, havendo necessidade absoluta de tratamento profilático. Eventualmente, o paciente pode procurar atendimento de urgência para tratamento de crises fortes. Uma série de terapêuticas, podem ser utilizadas para tratar a cefaléia em salvas Tabela VII.

Em alguns casos, o bloqueio do nervo occipital maior pode ser feito para aliviar crises de cefaléia em salvas, como já discutimos no tratamento da enxaqueca.

\subsection{Cefaléias secundárias}

O tratamento das cefaléias secundárias envolve, evidentemente, o tratamento da doença básica que a desencadeou (vide Capítulo I). Todavia, como todo fenômeno álgico leva a grande sofrimento, devemos tratar a dor. Para melhor podermos definir o tratamento, devemos classificar o tipo de dor que o paciente apresenta, e que responderá de forma diferente às várias terapêuticas possíveis. Na figura abaixo Figura 2 mostramos um algoritmo para tratamento das cefaléias secundárias.

\subsubsection{Classificação dos tipos de dor clínica}

Dor clínica pode ser classificada em dor nociceptiva e dor neuropática ${ }^{(28)}$.

\section{a) Dor nociceptiva}

a1) Somática - Ocorre como resultado da ativação de receptores nociceptivos, em tecidos cutâneos e profundos. É constante e bem localizada.

a2) Visceral - Origina-se da lesão de órgãos inervados pelo sistema nervoso simpático. Sua característica é vaga, e costuma ser mal locali-

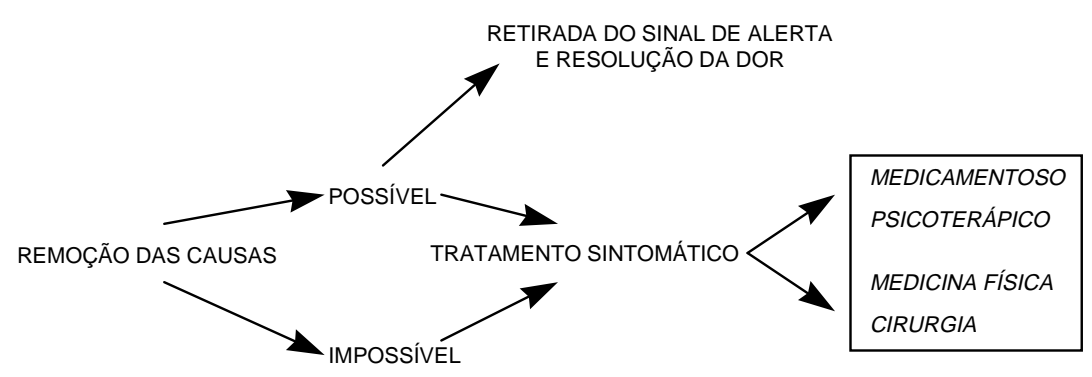

Figura 2 - Algoritmo para tratamento das cefaléias secundárias
Tabela VII - Terapêutica de cefaléia em salvas

- Inalação de Oxigênio a 100\% - 6-8 litros/minuto durante 10-20 minutos, com máscara, com paciente sentado. Cerca de $82 \%$ dos pacientes têm melhora da crise, se a inalação for feita no início. A resposta pode reforçar a impressão diagnóstica.

- Sumatriptan - devemos usar de preferência a formulação subcutânea da droga (6 mg), pelo fato de agir prontamente.

- Derivados do "ergot" - devemos usar formulações de ação rápida. Em nosso meio, contamos com alguns preparados comerciais que contêm 2 mg de Tartarato de Ergotamina, para uso sublingual. Nos países desenvolvidos, tem se utilizado o DHE (Diidroergotamina) por via parenteral (IM ou EV);

- Corticosteróides - são utilizados principalmente no tratamento profilático, porém, eventualmente, podem ser usados na unidade de emergência para tratamento de crises resistentes;

- Anestésicos locais - a aplicação tópica de Lidocaína na narina ipsilateral à crise dolorosa, anestesiando o gânglio esfenopalatino, pode, eventualmente, melhorar crises de cefaléia em salvas.

- Analgésicos (opiáceos)

zada. Muitas vezes, apresenta-se como "dor referida", na qual dor e hiperalgesia ocorrem em territórios superficiais, bem definidos, muitas vezes, distantes do sítio de afecção patológica. Estruturas profundas faciais, muitas vezes, determinam cefaléia com essas características. Assim, afecção de estruturas dentárias, muitas vezes, provocam dor, referida pelo paciente, em regiões temporais ou mesmo nucais.

\section{b) Dor neuropática}

Trata-se de dor associada com processos somatossensitivos aberrantes, induzidos por lesão de algum elemento do sistema nervoso. A dor neuropática pode ser central ou periférica. No caso da dor central, o sítio lesional é no sistema nervoso central. Assim, lesão em vias sensoriais no SNC (como no caso da lesão em nível talâmico), pode levar ao aparecimento de 
dores em território cefálico, principalmente na face. No caso de dor periférica, ela pode ocorrer em diversas condições: causalgia/distrofia simpaticorreflexa, dor das neuropatias periféricas, neuralgias, dores por compressão de tronco de nervo, formação de neuromas, membro fantasma. No território craniano, encontramos principalmente as neuralgias, dores por lesão de tronco nervoso periférico, dores por formação de neuromas. A dor neuropática acompanha-se de disestesias (desconforto e sensibilidade alterada aos estímulos normais, ocorrendo sensação de queimação, formigamento, adormecimento e prurido). Costuma ser constante e independente de estímulos evidentes. Hiperalgesia, alodinia e hiperpatia costumam ocorrer. Em tais casos há um caráter pelo qual denominamos dor por desaferentação. Eventualmente, ocorrem exacerbações da dor, que passam a ter caráter de choque, lancinante, de curta duração, tendo o que denominamos um caráter neurálgico. $\mathrm{O}$ caráter paroxístico define a dor como de caráter neurálgico.

\section{c) Dor psicogênica}

Existe muita controvérsia em relação à real existência da dor psicogênica. Embora todo fenômeno doloroso envolva um componente emocional, dores de caráter exclusivamente psíquico são extremamente infreqüentes. Todo paciente com dor deve ser considerado como acometido por lesão tissular, seja do tipo nociceptiva ou neuropática ou - o que não é infreqüente - com os dois componentes associados. A dor, como expressão exclusiva de doença psíquica - como expressão de fenômeno delusional - eventualmente ocorre, porém se trata de exceção absoluta. Alguns pacientes com dores cefálicas, principalmente dor facial atípica, apresentam tal quadro. Isso só confirma a necessidade, cada vez maior, de atendimento multidisciplinar, principalmente através de psicólogos e psiquiatras, aos pacientes com cefaléias de difícil controle.

\subsubsection{Terapêutica sintomática da dor cefálica}

Após o estabelecimento do diagnóstico do tipo de dor cefálica, utilizamos os esquemas terapêuticos relacionados a seguir ${ }^{(28)}$ :

\section{a) Dor nociceptiva}

- Analgésicos não opiáceos - salicilatos, derivados do indol (Indometacina), derivados do para-amino-fenol (Acetaminofeno), derivados do ácido antranílico (Ácido Mefenâmico), derivados do ácido arilpropiônico e drogas relacionadas (Naproxeno, Ibuprofeno, Cetoprofeno, Diclofenaco), derivados da pirazolona (Dipirona), oxicams (Piroxicam), nimesulida e outros. As doses e as vias de administração vão depender da situação clínica do paciente.

- Analgésicos opiáceos - agonistas (Morfina, Codeína, Meperidina ou Petidina, Dextropropoxifeno) e agonistas-antagonistas (Buprenorfina, Nalbufina, Tramadol, Pentazocina, Butorfanol).

- Corticosteróides - usados no tratamento da hipertensão intracraniana e da dor por invasão óssea de neoplasias. Eventualmente, podem ser usados para tratamento da dor por invasão de troncos nervosos.

\section{b) Dor neuropática}

Quando a dor neuropática tem um caráter mais do tipo desaferentação, utilizamos vários tipos de medicações, isolada ou associadamente: antidepressivos (Imipramina, Amitriptilina, Nortriptilina, Maprotilina, Trazodona, Mianserina, Fluoxetina), neurolépticos (Clorpromazina, Levomepromazina, Haloperidol, Tioridazina), antiespásticos (Baclofeno), gabaérgicos (Valproato de Sódio). Quando a dor neuropática tem caráter neurálgico - no território craniano, isto ocorre com certa freqüência, já que a maioria das neuralgias essenciais ocorre na face - utilizamos anticonvulsivantes (Carbamazepina, Oxcarbazepina, Difenilidantoína, Clonazepam, Valproato de Sódio) e, eventualmente, outras medicações (Baclofeno, Pimozide). Na unidade de emergência, em casos agudos e intensos de dores neurálgicas, temos utilizado a Difenilidantoína na forma endovenosa, com infusão de $18 \mathrm{mg} / \mathrm{kg}$, agudamente (não excedendo velocidade de infusão de $50 \mathrm{mg} /$ minuto), no sentido de atingir nível sérico de atuação mais rapidamente, e, posteriormente, utilizamos a dose de manutenção.

Seja na dor nociceptiva ou na neuropática, a utilização de outras formas de tratamento, psicoterápico, fisiátrico e/ou cirúrgico, pode, eventualmente, ser necessária.

\section{c) Dor psicogênica}

Evidentemente, em tais casos, o auxílio de profissional da área psiquiátrica é o aspecto mais importante. 
RABELLO GD. Clinical and therapeutic aspects of the acute headaches. Medicina, Ribeirão Preto, 30: 458-471, oct./dec. 1997.

ABSTRACT: The author reviews the clinical, diagnostic and therapeutic aspects of the acute headaches. Acute headache is a medical emergency and a correct and quick diagnosis are necessary in this situation because the success of the treatment depends on there conditions. The secundary headaches are frequent, although the primary headaches are important differential diagnosis.

UNITERMS: Headache. Migraine. Cluster Headache. Therapeutics.

\section{REFERÊNCIAS BIBLIOGRÁFICAS}

1 - BONICA JJ. Definitions and taxonomy of pain. In: The management of pain. Lea \& Febiger, Philadelphia, p. 18-27, 1990.

2 - PACKARD RC. What does the headache patient want? Headache 19: 370-374, 1979.

3 - ROWLINGSON JC. Classification and Assessment of chronic pain. In: RAJ PP, ed. Current review of pain, Current Medicine, Philadelphia, p. 37-46, 1994.

4 - FERRI-DE-BARROS JE \& NITRINI R. Que pacientes atende um neurologista? Alicerce de um currículo em Neurologia. Arq Neuropsiquiatr 54: 637-644, 1996.

5 - HEADACHE CLASSIFICATION COMMITTEE OF THE INTERNATIONAL HEADACHE SOCIETY - Classification and diagnostic criteria for headache disorders, cranial neuralgias and facial pain. Cephalalgia 8: 1-96, 1988. Suppl 7.

6 - SCHLAKE H-P et al. "Symptomatic migraine": Intracranial lesions mimicking migrainous headache - A report of three cases. Headache 31: 661-665, 1991.

7 - PAREJA JA et al. SUNCT Syndrome: Repetitive and overlapping attacks. Headache 34: 114-116, 1994.

8 - SJAASTAD O et al. Shortlasting, unilateral neuralgiform headaches attacks with conjunctival injection, tearing, sweating, and rhinorrhea. Cephalalgia 9: 147-156, 1989.

9 - GOADSBY PJ \& LIPTON RB. A review of paroxysmal hemicrânias, SUNCT syndrome and other short-lasting headaches with autonomic feature, including new cases. Brain 120: 193-209, 1997.

10 - PASCUAL J et al. Cough, exertional, and sexual headaches: An analysis of 72 benign and symptomatic cases. Neurology 46: 1520-1524, 1996.

11 - WEIR B. Headaches from aneurysms. Cephalalgia 14: 79-87, 1994.

12 - DAY JW \& RASKIN NH. Thunderclap headache: symptom of unruptured cerebral aneurysms. Lancet: 1247-1248, 1986.

13 - FORSYTH PA\& POSNER JB. Headaches in patients with brain tumors: a study of 111 patients. Neurology 43: 1678-1683, 1993.

14 - MATHEW NT; RAVISHANKAR K \& SANIN LC. Coexistence of migraine and idiopathic intracranial hypertension without papilledema. Neurology 46: 1226-1230, 1996.
15 - LAY CL; CAMPBELL JK \& MOKRI B. Low cerebrospinal fluid pressure headache. In: GOADSBY PJ \& SILBERSTEIN SD, eds. Headache, Butterworth-Heinemann, Boston, p. 355-367, 1997.

16 - PETERS GA \& HORTON BT. Headache with special reference of excessive use of ergotamine preparations and withdrawal effects. Proc Staff Meet Mayo Clin 26: 153-161, 1951.

17 - RABELLO GD. Cefaléia induzida por drogas. In: NITRINI R et al., eds. Condutas em Neurologia. Clínica Neurológica HC/FMUSP, São Paulo, p. 83-94, 1997.

18 - GOLDSTEIN J. Headache and acquired immunodeficiency syndrome. Neurol Clin 8: 947-960, 1990.

19 - SAHOTA PK \& DEXTER JD. Sleep and headache syndromes: A clinical review. Headache 30: 80-84, 1990.

20 - SJAASTAD O; FREDRIKSEN TA \& PFAFFENRATH V. Cervicogenic headache: Diagnostic Criteria. Headache 30: 725-726, 1990.

21 - CAPOBIANCO DJ. Facial pain as a symptom of nonmetastatic lung cancer. Headache 35: 581-585, 1995.

22 - BORDINI C et al. "Hemicrânia continua": A clinical review. Headache 31: 20-26, 1991.

23 - RASKIN NH. The Hypnic Headache Syndrome. Headache 28: 534-536, 1988.

24 - SOGES LJ et al. Migraine: evaluation by MR. Am J Neuroradiol 9: 425-429, 1988.

25 - PEROUTKA SJ. Dopamine and migraine. Neurology 49: 650-656, 1997.

26 - SWANSON JW \& WINNER P. Headache diagnosis and treatment in the emergency department. Course number 312, 49th Annual Meeting. American Academy of Neurology, Boston, p.1-11, 19-26 de abril, 1997.

27 - BASTOS GP et al. Uso da clorpromazina nas crises de cefaléia numa unidade de emergência (UE. Tema livre oral apresentado no XVII Congresso Brasileiro de Neurologia. Setembro/1996). Arq Neuropsiquiatr 54: O-076, 1996. Supl.

28 - RAJ PP. Characteristics, classification, and assessment of acute postoperative pain. In: RAJ PP, ed. Current review of pain. Current Medicine, Philadelphia, p. 17-35, 1994.

Recebido para publicação em 05/11/97

Aprovado para publicação em 10/12/97 